\title{
Insomnia among Active Patients with COVID-19: A Cross-Sectional Study
}

\author{
N.A. Uvais' ${ }^{1}$, Shamsudeen Moideen², Bishurul Hafi³ ${ }^{3}$ Sooraj Rajagopal4, \\ Maheshwari V ${ }^{5}$, and Tasneem Abdul Gafoor ${ }^{5}$ \\ Departments of ${ }^{1}$ Psychiatry, ${ }^{2}$ Internal Medicine, ${ }^{3}$ Dermatology, Iqraa International Hospital and Research Centre, Calicut, Kerala, India \\ ${ }^{4}$ Department of Physical Medicine and Rehabilitation, Government Medical College, Kozhikkode, India \\ ${ }^{5}$ Nandha College of Pharmacy, Erode, India
}

\begin{abstract}
Objective: Coronavirus disease (COVID-19) pandemic has become a source of abrupt disruptions in regular sleep habits (circadian rhythms) secondary to the associated severe physical and mental health issues. However, there are no studies, to the best of our knowledge, providing evidence based information on sleep-related disorders among patients with COVID-19 from India till date. In the present cross sectional studies, we explored the prevalence of insomnia and its correlations in a COVID-19 patient population in South India. Methods: A cross-sectional study was conducted among patients admitted with COVID-19 in a designated COVID-19 treatment centre. Sociodemographic and clinical details were collected according to a structured performa. Medical records were reviewed to collect information about medical background, overall medical parameters of COVID-19, laboratory values and treatment details. The insomnia was evaluated using the Insomnia Severity Index. Results: The mean age of the participants was 51.59 years, and 73 (53.7\%) participants were females. Twenty-three (16.9\%) participants had subthreshold insomnia, and $3(2.2 \%)$ participants had clinical insomnia. The white blood cell count was higher in the insomnia group $\left(8.5 \times 10^{9} / \mathrm{L}\right)$ than in the non-insomnia group $\left(6.4 \times 10^{9} / \mathrm{L}\right)(\mathrm{p}=0.003)$. The lymphocyte count was significantly lower in the insomnia group (24.13\%WB) compared with the non-insomnia group ( $31.41 \% \mathrm{WB})(\mathrm{p}=0.008)$. The ALT, AST, and serum creatinine levels were significantly higher among the insomnia group. Conclusion: Insomnia may occur among active patients with COVID-19 infection. It is important to keep in mind that there is a hidden danger of the transformation of acute insomnia into chronic insomnia among COVID-19 patients. It is important to disseminate sleep health education and sleep hygiene behavior interventions to the COVID-19 patient population while they are admitted in the hospital.
\end{abstract}

Key Words: Insomnia; COVID-19; Inpatients; India

Received: January 19, 2021 Revised: March 5, 2021 Accepted: March 7, 2021

Corresponding author: Bishurul Hafi, MD, Department of Dermatology, Iqraa International Hospital and Research Centre, Calicut, Kerala, India.

Tel: 91-7085740869, E-mail: bishuru@gmail.com

(a) This is an Open Access article distributed under the terms of the Creative Commons Attribution Non-Commercial License (https://creativecommons.org/licenses/bync/4.0) which permits unrestricted non-commercial use, distribution, and reproduction in any medium, provided the original work is properly cited.

\section{INTRODUCTION}

Coronavirus disease (COVID-19), a highly contagious virus, first observed in the Chinese city of Wuhan in late December of 2019, has spread across the world resulting in significant morbidity and mortality [1]. The nature of the pandemic with respect to lack of any specific treatment or vaccine along with strict control measures has resulted in significant psychological symptoms among general population [2]. Moreover, patients with COVID-19 infection also been shown to be vulnerable to a various psychological disorders even after they have been cured and discharged from hospital [3]. Currently, there are only few epidemi- ological studies available on the mental health problems and psychiatric morbidity of those diagnosed with COVID-19. Moreover, COVID-19 related mental health research is scarce in lower-middle-income countries of Asia [4].

When facing the COVID-19 infection, a normal sleep pattern is essential due to its many benefits for mental and physical health [5]. Lack of sleep can result in various negative outcomes including poor immune response [5]. COVID-19 pandemic has become a source of abrupt disruptions in regular sleep habits (circadian rhythms) secondary to the associated severe physical and mental health issues [6]. However, there are no studies, to the best of our knowledge, providing evidence based information on sleep- 
related disorders among patients with COVID-19 from India till date. In the present cross sectional studies, we explored the prevalence of insomnia and it's correlates in a COVID-19 patient population in South India.

\section{METHODS}

It was a cross-sectional study conducted at a COVID-19 treatment facility managed by public-private partnership in South India. The study was approved by the hospital ethical committee. The study period was 1st August 2020 to 30th September 2020. Purposive sampling was used to recruit participants. Inclusion criteria was COVID-19 patients above 18 years of age, who were clinically stable for the interview. Informed consent was taken from all the participants. Sociodemographic and clinical details were collected according to a structured performa. Medical records were reviewed to collect information about medical background, overall medical parameters of COVID-19, laboratory values and treatment details. The insomnia was evaluated using the Insomnia Severity Index (ISI). ISI is a 7-item scale that measures the self-reported level of sleep quality and insomnia [7]. Insomnia on the ISI was classified as none (0-7), sub-threshold (8$14)$, moderate (15-21), or severe (22-28).

Data were analyzed with the SPSS Version 23.0 (IBM Corp., Armonk, NY, USA). Continual variables were expressed as mean \pm standard deviation. Categorical variables were expressed as frequencies and percentages. An independent-sample t-test was used to test for differences in continuous variables between COVID-19 patients with insomnia and those without insomnia. Differences were considered as statistically significant if the $p$ value was $<0.05$.

\section{RESULTS}

Among 136 participants, 24 (17.6\%) and 30 (22.1\%) were in the age group of 11-30 and 31-50, respectively, and $82(60.3 \%)$ were above 50 years. The mean age was 51.59 years, and 73 (53.7\%) participants were females. Twenty-two (16.2\%) patients and 25 (18.4\%) had hospital and family exposure, respctively, and 89 (65.4\%) had unknown exposure. Ninty-one (67.4\%) participants were having symptoms of COVID-19, and 93 (73.2\%) had comorbid medical illness. Twenty-three (16.9\%) participants had subthreshold insomnia, and 3 (2.2\%) participants had clinical insomnia. The sociodemographic and clinical details are summarised in Table 1.

After comparing the white blood cell (WBC) counts between COVID-19 patients with and without insomnia, results showed that the WBC count was higher in the insomnia group $\left(8.5 \times 10^{9} / \mathrm{L}\right)$ than in the non-insomnia group $\left(6.4 \times 10^{9} / \mathrm{L}\right)(\mathrm{p}=0.003)$. The lymphocyte count was significantly lower in the insomnia group $(24.13 \%$ $\mathrm{WB})$ compared with the non-insomnia group $(31.41 \% \mathrm{WB})(\mathrm{p}=$ 0.008). The alanine aminotransferase (ALT), aspartate transaminase (AST), and serum creatinine levels were significantly high-
Table 1. Sociodemographic and clinical variables

\begin{tabular}{lc}
\multicolumn{1}{c}{ Variable } & Value $(\mathrm{n}=136)$ \\
Age & \\
$11-30$ years & $24(17.6)$ \\
$31-50$ years & $30(22.1)$ \\
$51-70$ years & $66(48.5)$ \\
$>70$ years & $16(11.8)$ \\
Sex & \\
Female & $73(53.7)$ \\
Male & $63(46.3)$ \\
Exposure & \\
Hospital & $22(16.2)$ \\
Family & $25(18.4)$ \\
Unknown & $89(65.4)$ \\
Symptoms & \\
Asymptomatic & $44(32.6)$ \\
Symptomatic & $91(67.4)$ \\
Comorbidities & \\
Yes & $93(73.2)$ \\
No & $34(26.8)$ \\
Insomnia & \\
Nil & $110(80.9)$ \\
Subthreshold & $23(16.9)$ \\
Clinical & $3(2.2)$ \\
\hline
\end{tabular}

Data are presented as number (\%).

Table 2. Comparison of blood investigation parameters in COVID-19 patients with and without insomnia

\begin{tabular}{lrrr}
\multicolumn{1}{c}{ Variable } & \multicolumn{1}{c}{ No } \\
insomnia & Insomnia & p value \\
Haemoglobin $(\mathrm{mg} / \mathrm{dL})($ mean) & 12.53 & 12.13 & 0.400 \\
WBC $\left(\times 10^{9} / \mathrm{L}\right)($ mean) & 6.40 & 8.50 & 0.003 \\
Neutrophil, \%WB (mean) & 59.83 & 64.50 & 0.151 \\
Lymphocyte, \%WB (mean) & 31.41 & 24.13 & 0.008 \\
Monocyte, \%WB (mean) & 6.75 & 5.50 & 0.155 \\
Platelet count $\left(\times 10^{9} / \mathrm{L}\right)($ mean) & 2.77 & 2.84 & 0.867 \\
ALT (units/L) & 26.06 & 40.74 & 0.006 \\
AST (units/L) & 24.85 & 35.00 & 0.070 \\
Serum creatinine $(\mathrm{mg} / \mathrm{dL})$ & 1.07 & 2.06 & 0.015 \\
D-dimer $(\mathrm{ng} / \mathrm{mL})$ & 1452.47 & 1195.59 & 0.808 \\
\hline
\end{tabular}

Data are mean values. Insomnia if a score is $>7$ in the Insomnia Severity Index (ISI) questionnaire. WBC: white blood cell, ALT: alanine aminotransferase, AST: aspartate transaminase

er among the insomnia group. The details are summarised in the Table 2.

\section{DISCUSSION}

To the best of our knowledge, the current study is one of the few to present data on the mental health comorbidity (insomnia) among patients with active COVID-19 infection. The overall prevalence of insomnia among 136 COVI-19 patients is $19.1 \%$. A recent meta-analysis exploring sleep problems during COV- 
ID-19 pandemic by population found that COVID-19 patients were the most affected group with sleep problems, with a pooled rate of $74.8 \%$, when compared with general population $(32.3 \%)$ and healthcare workers (36.0\%) [5]. The significantly lower prevalence of sleep problems in our study can be explained on the basis of the scale used. Most of the studies which reported higher prevalence of sleep problems among COVID-19 patients were done using the Pittsburgh Sleep Quality Index (PSQI), which captures a very wide range of sleep-related issues such as nightmares, snoring, sleep medication use etc. Recent studies using ISI or other researcher-developed measures to quantify sleep problems reported a significantly lower prevalence of sleep problems (25.2\%) in the above meta-analysis [5]. However, a recent meta-analysis comparing diagnostic accuracy of PSQI and ISI indicated that summary estimates of sensitivity and specificity did not differ significantly between the two measures [8]. Insomnia among COVID-19 patients might be due to the stress associated with infection as worry provokes uncontrollable cognitive arousal which is one of the major mechanisms inducing dysregulation in sleep [9]. However, it can also be partly attributed to symptoms and side effects of medications [5].

We found significantly higher WBC count in the insomnia group than in the non-insomnia group. However, considering the previous literature regarding changes in the WBC count following short periods of insomnia, it might also be the result of insomnia rather than the higher inflammatory response in the COVID-19 positive insomnia group. Boudjeltia et al. [10] explored the effects of sleep restricted to four hours for three consecutive nights on blood parameters and found that a significant increase in $\mathrm{WBC}\left(5.79 \times 10^{3}\right.$ vs. $6.89 \times 10^{3}$ cells $\left./ \mu \mathrm{L}, \mathrm{p}=0.03\right)$, and neutrophils $\left(3.17 \times 10^{3}\right.$ vs. $4.24 \times 10^{3}$ cells $\left./ \mu \mathrm{L}, \mathrm{p}=0.01\right)$ was observed after the third night of sleep restriction. Moreover, Nishitani and Sakakibara [11] explored the relation between sleep difficulty and WBC count in shift workers and found that higher WBC counts were more frequently found in shift workers with sleeping problems than those without the problems.

We also found that lymphocyte count was significantly lower in the insomnia group when compared to the non-insomnia group. Among the patients with COVID-19, lymphopenia has been shown to be associated with adverse outcome, intensive care unit stay, higher risk of myocardial injury and increased risk of acute respiratory distress syndrome [12]. The reason for this phenomenon was thought to be due to the the presence of ACE2 receptor on their surface and SARS-CoV-2 may directly infect those cells and ultimately lead to their lysis [12].

We also found significantly higher levels of liver enzymes (AST, ALT) among COVID-19 patients with insomnia when compared to non-insomnia group. Recent evidence also suggest that that the circadian clock can alter the levels of AST and ALT [13]. Another study found that the continuous sleep deprivation from $76 \mathrm{~h}$ to $80 \mathrm{~h}$ resulted in an increase of $170 \%$ in mean AST, and $58-5 \%$ in mean ALT [14]. Insmonia symptoms can be a manifestation of underlying depression [15].
In conclusion, insomnia may occur among active patients with COVID-19 infection. Patients with COVID-19 with insomnia have significantly higher WBC count, AST, ALT, and serum creatinine, and significantly lower lymphocyte count, when compared to the non-insomnia group. It is important to keep in mind that there is a hidden danger of the transformation of acute insomnia into chronic insomnia among COVID-19 patients. Insomnia symptoms should alert physicians about possible depression which is very frequent and one of the core symptoms. It is important to disseminate sleep health education and sleep hygiene behavior interventions to the COVID-19 patient population while they are admitted in the hospital.

\section{Acknowledgments}

None

\section{Conflicts of Interest}

The authors have no potential conflicts of interest to disclose.

\section{Author Contributions}

Conceptualization: N.A. Uvais. Data curation: Shamsudeen Moideen. Formal analysis: N.A. Uvais. Investigation: Shamsudeen Moideen, Maheswari V, Tasneem Abdul Gafoor. Methodology: N.A. Uvais. Project administration: Bishurul Hafi. Resources: N.A. Uvais, Maheswari V. Software: N.A. Uvais. Supervision: Bishurul Hafi. Validation: Shamsudheen Moideen, Sooraj Rajagopal. Visualization: N.A. Uvais. Writing_original draft: N.A. Uvais, Bishurul Hafi. Writing—review \& editing: all authors.

\section{ORCID iDs}

\section{N.A. Uvais (1)}

https://orcid.org/0000-0003-0961-3025

Shamsudeen Moideen (1)

https://orcid.org/0000-0002-4362-0424

Bishurul Hafi (1)

https://orcid.org/0000-0001-9749-8906

Sooraj Rajagopal (1)

https://orcid.org/0000-0002-3375-1527

\section{REFERENCES}

1. Borges do Nascimento IJ, Cacic N, Abdulazeem HM, von Groote TC, Jayarajah U, Weerasekara I, et al. Novel coronavirus infection (COVID-19) in humans: a scoping review and meta-analysis. J Clin Med 2020;9:941.

2. Huang Y, Zhao N. Generalized anxiety disorder, depressive symptoms and sleep quality during COVID-19 outbreak in China: a web-based cross-sectional survey. Psychiatry Res 2020;288:112954.

3. Cai X, Hu X, Ekumi IO, Wang J, An Y, Li Z, et al. Psychological distress and its correlates among COVID-19 survivors during early convalescence across age groups. Am J Geriatr Psychiatry 2020;28:1030-1039.

4. Kar SK, Oyetunji TP, Prakash AJ, Ogunmola OA, Tripathy S, Lawal MM, et al. Mental health research in the lower-middle-income countries of Africa and Asia during the COVID-19 pandemic: a scoping review. Neurol Psychiatry Brain Res 2020;38:54-64.

5. Jahrami H, BaHammam AS, Bragazzi NL, Saif Z, Faris M, Vitiello MV. Sleep problems during the COVID-19 pandemic by population: a systematic review and meta-analysis. J Clin Sleep Med 2021;17:299-313. 
6. Kokou-Kpolou CK, Megalakaki O, Laimou D, Kousouri M. Insomnia during COVID-19 pandemic and lockdown: prevalence, severity, and associated risk factors in French population. Psychiatry Res 2020;290:113128.

7. Morin CM, Belleville G, Bélanger L, Ivers H. The Insomnia Severity Index: psychometric indicators to detect insomnia cases and evaluate treatment response. Sleep 2011;34:601-608.

8. Chiu HY, Chang LY, Hsieh YJ, Tsai PS. A meta-analysis of diagnostic accuracy of three screening tools for insomnia. J Psychosom Res 2016;87:85-92.

9. Kalmbach DA, Cuamatzi-Castelan AS, Tonnu CV, Tran KM, Anderson JR, Roth T, et al. Hyperarousal and sleep reactivity in insomnia: current insights. Nat Sci Sleep 2018;10:193-201.

10. Boudjeltia KZ, Faraut B, Stenuit P, Esposito MJ, Dyzma M, Brohée D, et al. Sleep restriction increases white blood cells, mainly neutrophil count, in young healthy men: a pilot study. Vasc Health Risk Manag 2008;4:1467-1470.

11. Nishitani N, Sakakibara H. White blood cell count and sleep difficulty ex- amined by the Athens Insomnia Scale in shift workers. Open Sleep J 2010; $3: 1-5$.

12. Fan BE, Chong VCL, Chan SSW, Lim GH, Lim KGE, Tan GB, et al. Hematologic parameters in patients with COVID-19 infection. Am J Hematol 2020;95:E131-E134.

13. Li T, Cao R, Xia R, Xia Z. Effects of 72 hours sleep deprivation on liver circadian clock gene expression and oxidative stress in rats. Yangtze Medicine 2017;1:194-201.

14. Ilan Y, Martinowitz G, Abramsky O, Glazer G, Lavie P. Prolonged sleep-deprivation induced disturbed liver functions serum lipid levels, and hyperphosphatemia. Eur J Clin Invest 1992;22:740-743.

15. Geoffroy PA, Hoertel N, Etain B, Bellivier F, Delorme R, Limosin F, et al. Insomnia and hypersomnia in major depressive episode: prevalence, sociodemographic characteristics and psychiatric comorbidity in a populationbased study. J Affect Disord 2018;226:132-141. 